Research Article

\title{
Prescription audit of corticosteroid usage in the department of dermatology at a tertiary care teaching hospital
}

\author{
Mirshad PV, Afzal Khan AK*, Fasalu Rahiman OM, Mohammed Muneersha TK
}

Department of Pharmacology, MES Medical College, Kerala679338, India

Received: 11 May 2013

Accepted: 20 May 2013

*Correspondence to:

Dr. Afzal Khan AK,

Email:

drafzalkhan4u@gmail.com

C 2013 PV Mirshad et al. This

is an open-access article distributed under the terms of the Creative Commons

Attribution License, which permits unrestricted use, distribution, and reproduction in any medium, provided the original work is properly cited.

\begin{abstract}
Background: Corticosteroids are a group of commonly used drugs in dermatology practice whose non judicious use frequently results in undesirable and unwanted effects. Prescribing them rationally with care allows us to derive the maximum benefit out of them with minimal side effects.

Methods: Retrospective analysis of 112 case files belonging to patients admitted in the department of dermatology over a period of one year was undertaken to ascertain the usage pattern of corticosteroids in relation to their potency, strength, frequency, duration, route, quantity to be applied. The data thus collected was expressed in terms of averages, ratios and proportions.

Results: The total number of formulations prescribed were 929 out of which $10.8 \%$ were corticosteroids. The average number of formulations prescribed per patient was 8.29. About $78(70 \%)$ patients admitted in dermatology received corticosteroids. Topical steroids were prescribed in 50 patients $(44.7 \%)$ out of whom $36(72 \%)$ received only topical, the remaining $14(28 \%)$ were prescribed both systemic and topical corticosteroids.

Brand names were used in all cases. Highly potent corticosteroids like clobetasol, halobetasol and mometasone were prescribed to $39(50 \%)$ of all cases who received topical corticosteroids.

Conclusion: The study reveals the deficiencies which exist in the present prescribing pattern of corticosteroids. Educational interventions among the doctors as well as students should be carried out to in order to promote rational drug use.
\end{abstract}

Keywords: Prescription analysis, Dermatology, Drug utilization, Corticosteroids, Rational drug use

\section{INTRODUCTION}

Corticosteroids or glucocorticoids, which were introduced in the late 1950s, had brought about a revolution in the treatment of dermatological diseases and even to this day they constitute one of the largest and commonly used groups of drugs in dermatological practice. ${ }^{1}$

These highly effective drugs are, on one hand, of great advantage in certain indications, localizations, and types of disease; however, they may produce deleterious effects when used over longer periods or when not properly utilized. $^{2}$

The amount of corticosteroid which is prescribed, dispensed and applied should be considered carefully because too little steroid can lead to a poor response, and too much can increase the risk of adverse effects. ${ }^{3}$
Rational use can minimise the systemic and cutaneous side effects associated with corticosteroids. ${ }^{4}$ In order to derive the optimum benefit with least adverse effects, various factors have to be taken into consideration while prescribing, including the nature of the disease, age of the patient, site affected, and the pharmacology of the corticosteroids like potency, frequency of use and the vehicle. $^{5}$

Intermittent monitoring of drug use patterns is one of the measures which is undertaken in order to analyze the rationality of drug usage and to offer feedback/ suggestions to the prescribers.

The main aim of drug utilization research is to facilitate the rational drug use. Without a precise knowledge of how drugs are being prescribed and used, it is difficult to suggest measures to improve the prescribing habits. ${ }^{7}$ 
Hence by undertaking the present study we sought to study the various parameters related to the use of corticosteroids in the patient population so as to identify any deficiencies in their pattern of use so that effective interventions or modifications can be suggested if necessary.

\section{METHODS}

Case files belonging to 112 patients admitted in the department of dermatology of a tertiary care teaching hospital in Kerala, over a period of one year were retrieved with the assistance of the medical records department.

A detailed retrospective analysis was performed by noting down the details of the prescription chart. Patient related information including age, sex, disease, days of hospital stay was collected. Information related to the use of corticosteroids such as name of the drug, number of drugs, dosage form, dosing frequency, duration, route of administration, quantity to be used or applied and potency were also recorded.

The data was analysed using descriptive statistics. Ratios, proportions and percentages were used to describe the data.

\section{RESULTS}

The average age of the 112 patients whose case files were analysed was 47.07 years. Majority of the patients (52\%) were above the age of 51 years. Only 13(11.6\%) of the patients were less than 25 years of age.

Papillosquamous diseases including psoriasis were found to be the most common cause for admission (29.5\%) followed by eczematous diseases like contact, seborrheic and atopic dermatitis (17.9\%).

The total number of formulations prescribed for the 112 inpatients admitted in the dermatology ward were 929, i.e. a mean of 8.29 per patient. Out of which 101 preparations consisted of corticosteroids which were prescribed to $78 / 112(70 \%)$ patients at an average of 1.29 preparations per patient.

57 of these formulations consisted of topical corticosteroids, 34 were parenteral corticosteroids and 10 were oral corticosteroid preparations. All (100\%) of these were prescribed by their trade/brand names.

Most of the patients receiving corticosteroids were prescribed a topical corticosteroid preparation i.e. $64 \%$ (50/78), out of which 13 patients were also administered a systemic, oral or parenteral corticosteroid preparation in addition to the topical agent and seven patients were prescribed two topical corticosteroid preparations at the same time.
Fixed dose combinations (FDCs) consisted of $47.5 \%$ $(48 / 101)$ of the formulations which were prescribed and all the prescribed FDCs were topical. The most common FDCs prescribed along with a corticosteroid preparation were gentamicin (19/48) followed by salicylic acid (12/48), fusidic acid (11/48) and miconazole (3/48).

Frequency of administration and the strength of the medication were clearly entered for all systemic corticosteroid preparations but in case of topical only two prescriptions mentioned these parameters. Duration of treatment was mentioned for almost all systemic and topical preparations in 93\% (96/101) of the prescriptions. The quantity of medication to be applied topically was mentioned in only one prescription.

Among the topical preparations prescribed 78\% (39/50) were highly potent corticosteroids including clobetasol, halobetasol and mometasone. Intermediate potency ones like hydrocortisone, Fluticasone and betamethasone consisted of $18 \%(9 / 50)$ and the remaining $4 \%(2 / 50)$ consisted of a less potent dexamethasone preparation. Systemically dexamethasone and prednisolone were used most commonly i.e. $86 \%(38 / 44)$.

A few prescriptions for systemic corticosteroids (14/44) specifically carried the instruction of administering the medication in the morning.

\section{DISCUSSION}

Since corticosteroids are one of the commonest groups of drugs used in dermatological practice an assessment of their usage pattern in order to encourage their proper and rational utilization is commonly recommended in order to optimize their benefits and limit their adverse effects.

The findings of our study correlate with the finding of earlier studies carried out in India and elsewhere wherein potent topical corticosteroids were commonly used in $43 \%$ patients and the quantity of topical steroid was mentioned for only $4 \%$ of patients. ${ }^{8}$ Not specifying the quantity can result in under-usage of the preparation causing subsequent sub-therapeutic outcome, at the same time excessive usage can result in unwanted effects. ${ }^{1,3}$

Clear explanations should be given, so that patients are aware of how much steroid to use, where and when to apply it, and for how long. The risk of side-effects increases with steroid potency and the amount used. ${ }^{9}$

Emollients are underused inspite of the evidence of their 'steroid sparing effect' i.e. their ability to reduce the need to use topical steroids especially in patients with atopic eczema and psoriasis. ${ }^{9}$ 
Systemically dexamethasone was commonly prescribed to most of the patients which may be due to its potency and longer duration of action. , $^{6}$

In all our prescription charts the medications were prescribed by their brand names, generic names were never preferred which was also the case in a previous study conducted in Palestine. ${ }^{1}$ Use of generic names usually provides flexibility to the dispensing pharmacist and generic drugs are less expensive than brand-name drugs. 11

Another concern was the use of FDCs of topical steroids with an antimicrobial agent. Studies have shown that topical antimicrobial/steroid combinations do not confer any benefit over steroids alone in patients with atopic eczema. $^{9}$

Topical antibiotics should only be used where infection is limited to a small area of the skin. A short course of a suitable oral antibiotic may be indicated in more severe cases. To prevent the development of resistance, all antimicrobials, including topical agents, should be used very judiciously. ${ }^{9}$

\section{CONCLUSION}

The findings of our study show the usage of highly potent topical corticosteroids. Another common finding was the inadequate prescribing information especially regarding the quantity of topical medication to be applied and the excessive use of brand names.

Repeated monitoring of drug usage combined with regular feedback to the prescribers is essential. Measures to facilitate and encourage rational prescribing among the students and prescribers should be undertaken.

Funding: No funding sources Conflict of interests: None declared Ethical approval: Not Required

\section{REFERENCES}

1. Sweileh WM. Audit of prescribing practices of topical corticosteroids in outpatient dermatology clinics in north Palestine. Eastern Mediterranean Health Journal 2006;12:161-9.

2. Schoepe S, Schacke H, May E, Asadullah K. Glucocorticoid therapy-induced skin Atrophy. Exp Dermatol 2006:15:406-20.

3. Ference JD, Last AR. Choosing Topical Corticosteroids. Am Fam Physician 2009;79:13540.

4. Sharma SC, Uppal R, Sharma PL, Kaur S. Rational Use of Topical Corticosteriods in Dermatology. Indian J Pharmacol 1990;22:141-4.

5. Lee M, Marks R. The role of corticosteroids in dermatology. Aust Prescr 1998;21:9-11.

6. Ashok Kumar M, Noushad PP, Shailaja K, Jayasutha J, Ramasamy C. A Study on drug prescribing pattern and use of corticosteroids in dermatological conditions at a tertiary care teaching hospital. International Journal of Pharmaceutical Sciences Review and Research 2011;9:132-5.

7. WHO. Introduction to Drug Utilization Research. Geneva. 2003.

8. Uppal R, Sharma SC, Bhowmik SR, Sharma PL, Kaur S. Topical corticosteroids usage in dermatology. Int J Clin Pharmacol Ther Toxicol 1991;29:48-50.

9. The National Prescribing Centre. Using topical corticosteroids in general practice. MeReC Bulletin 1999;10(6):21-4.

10. Ankit P, Bharat G. Study of drug utilization pattern of glucocorticosteroid drugs with special emphasis on their immediate adverse effects in a tertiary care teaching rural hospital. Indian Journal of Pharmacy Practice 2010;3:18-23.

11. Oshikoya KA, Bello JA, Ayorinde EO. Prescribing knowledge and skills of final year medical students in Nigeria. Indian J Pharmacol 2008;40,25:51-5.

doi:10.5455/2319-2003.ijbcp20130813

Cite this article as: PV Mirshad, AK Afzal Khan, OM Fasalu Rahiman, TK Mohammed Muneersha. Prescription audit of corticosteroid usage in the department of dermatology at a tertiary care teaching hospital. Int J Basic Clin Pharmacol 2013;2:411-3. 